\title{
TRANSANNULAR INTERACTIONS IN [2.2] PHANES AS STUDIED BY MAGNETIC RESONANCE AND OPTICAL SPECTRA
}

\author{
D. SCHWEITZER, J.P. COLPA ${ }^{\star}$, J. BEHNKE * , K.H. HAUSSER \\ Mor-Planck-Institute, Department of Molecular Physics, 6900 Heidelberg, W. Germany
}

and

M. HAENEL and H.A. STAAB

Heidelberg University, Institute of Organic Chemistry, 6900 Heidelberg. W. Germany

Received 16 April 1975

The interaction of $\pi$-electrons in [2.2] phanes was studied both experimentally and theoretically. The fluorescence and phosphorescence spectra were measured at liquid helium temperature; in addition, the zero field splitting parameters $D$ and $E$ were determined by ODMR in zero field and by ordinary ESR at X-band. The results for the phanes with two identical aromutic units can be summarized as follows: The rather small reduction of the $D$ and $E$ values of the order of $10 \%$ with respect to the monomers indicates, in agreement with the theoretical treatment given in part II, that the two unpaircd electrons of the excited triplet state have a high probability to be at a given time in the same half of the molecule. While the fluorescence spectra show the typical behaviour of emission spectra of dimers or excimers, the phosphorescence spectra cxhibit some remaining structure. This behaviour which indicates a somewhat weaker coupling among the triplet orbitals as compared to the singlet orbitals can also be understood on the basis of thcoretical considerations. For a phane with two different aromatic units the behaviour is found to be more similar to the corresponding aromatic monomer with the lower excited states with some perturbation by the other part of the phane also in agreenent with theoretical expectation.

\section{Introduction}

The mutual interaction between $\pi$-electrons of two aromatic molecules which are close to one another may result in changes of the relative positions of the energy levels of the various electronic states. Förster and Kasper [1,2] first observed in the emission spectrum of pyrene in solution a broad structureless fluorescence which was shifted to longer wavelength as compared to the normal vibrationally structured band. They suggested that this broad band fluorescence originates from an excited dimer molecule, in which an excited molecule is associated with a second molecule in the ground state. Stevens [3] termed this phenomenon an "excimer" in distinction to dimers which are

\footnotetext{
- On leave from: Queen's University, Department of Chemistry, Kingston, Ontario, Canada.

*t Present address: Pharma-Gummi GmbH Eschweiler-
}

stable in the ground state as well as in the excited state.

Subsequently, excimers were observed in concentrated solutions [4-7], pure liquids [8], and crysta]s $[9,10]$ for a large number of aromatic hydrocarbons. In addition to the broad band fluorescence, a similar excimer phosphorescence was found $[11,12]$.

Another class of compounds where interaction of $\pi$-electron clouds through space can easily be studied are the paracyclophanes [13] and other phanes [14-16] in which two aromatic units are kept in a face-to-face arrangement by methylene bridges. Luminescence and absorption was studied by El-Sayed [17], Rice and co-workers [18-20] and more recently Nagakura and co-workers $[21,22]$ for the 1 iracyclophanes and by Froines et al. [23] for the [2.2] $(1,4)$ naphthalenophanes. The spectra show typical excimer emissions and are explained by configuration interac. tion of neutral exciton states and charge transfer states $[19,20]$. 
Beside the changes of energy differences also the possible changes of magnetic properties, such as spin distribution in the triplet state, are of interest. So Bramwell et al. [24] and Haenel [25] studied the influence of such interacting aromatic systems on the root mean square zero field splitting parameter $D^{*}$ by normal ESR methods in randomly oriented samples. The zero field splitting parameters $D$ and $E$ could not be measured with the exemption of a multi-layered paracyclophane [24]. Nevertheless, one important conclusion was made: The model of the triplet state of [2.2] phanes with one unpaired electron in each half of the sandwich compound seems to be very unlikely [25].

In this paper we have studied the emission spectra and the zero field splitting parameters $D$ and $E$ of the following phanes which are shown in fig. 1: anti-[2.2]$(1,4)$ naphthalenophane $(4),[2.2](2,6)$ naphthalenophane (5), [2.2] $\left(4,4^{\prime}\right)$ biphenylophane (8), 19, 20-dihydro[2] (4.4')biphenylo [2](2,7)phenanthrenophane (9), 5,6,17, 18-tetrahydro [2.2] $(2,7)$ phenanthrenophane (10), [2.2] $(2,7)$ phenanthrenophane $(13 / 14)$, and $[2]\left(4,4^{\prime}\right)$ biphenylo $[2](2,7)$ phenanthrenophane (15).

The $D$ and $E$ values were measured by optical detection of magnetic resonance (ODMR) in zero field as well as in sone cases with normal ESR in X-band in randomly oriented simples. The results are interpreted in terms of a theoretical molecular orbital treatment of such double molecules which is given in section 2 .

\section{Excited triplet and singlet states of double molecules and phanes}

Suppose we have two identical planar aromatic molecules, in such a position that their molecular planes are parallel and that the two molecules are each other's mirror image with respect to a plane parallel to the molecular planes and equidistant to both of them. We take the molecular planes in vertical position and we distinguish the two molecules as a left and a right one. When we consider the two molecules at a sufficiently large distance as to be completely decoupled, we may describe their ground and excited states completely independently. One call consider the two molecules as one system, however, and come to essentially the same description of the states, as will be
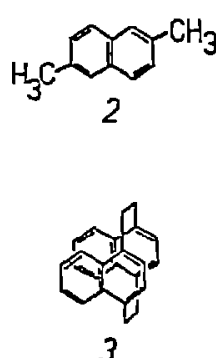

3

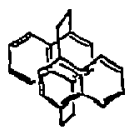

4

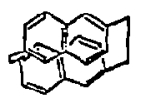

5
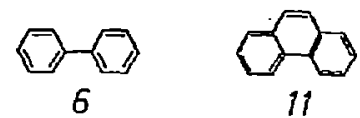

11

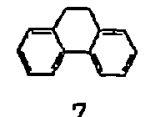

7

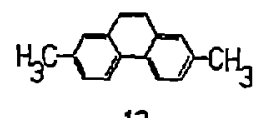

12

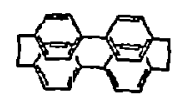

8
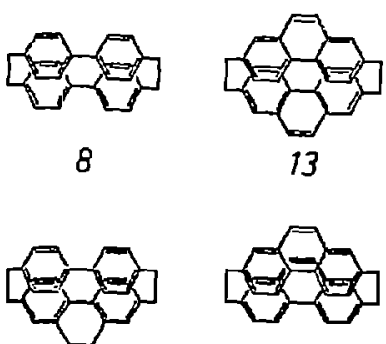

9

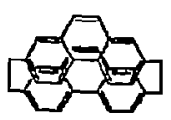

14

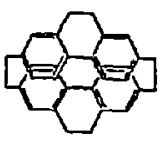

10

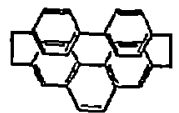

15

Fig. 1. [2.2] phanes and corresponding monomers: naphthalene (1); 2, 6-dimethylnaph thalene (2); syn-[2.2] $(1,4)$ naphthalenophane (3); anti-[2.2] $(1,4)$ naphthalenophane (4); $[2.2](2,6)$ naphthalenophane (5); biphenyl (6); 9, 10-dihydro-phenanthrene (7); [2.2] (4.4') biphenylophane (8); 19,20-dihydro [2]$\left(4,4^{\prime}\right)$ biphenylo $[2](2,7)$ phenanthrenophane $(9) ; 5,6,17,18$ tetrahydro[2.2] (2.7)phenanthrenophane (10); phenanthrene (11); 2,7-dimethylphenanthrene (12); $[2.2](2,7)$ phenenthrenophane $(13,14) ;[2]\left(4,4^{\prime}\right)$ biphenylo $[2](2,7)$ phenanthrenophane (15).

shown in this section.

In order to simplify the notation, we consider only the highest occupied $\pi$ orbitals $L$ and $R$ and the lowest unoccupied $\pi^{*}$ orbitals $l$ and $r$ for the left and the right hand molecule, respectively. The orbitais $L$ and $R$ are degenerate and so are the orbitals $l$ and $r$ because the two molecules are identical. When we consider the two molecules at large separation as one system, we get for a delocalized $\mathrm{MO}$ description the new orbitals $2^{-1 / 2}(L+R) ; 2^{-1 / 2}(L-R) ; 2^{-1 / 2}(l+r)$ and $2^{-1 / 2}(l-r) ;$ the orbitals $2^{-1 / 2}(L+R)$ and $2^{-1 / 2}(l+r)$ are antisymmetric with respect to the symmetry plane between the molecular planes; the other two are sym- 
metric with respect to this plane. When the two molecules are uncoupled, the orbitals $2^{-1 / 2}(L+R)$ and $2^{-1 / 2}(L-R)$ are degenerate; so are the orbitals $2^{-1 / 2}(l+r)$ and $2^{-1 / 2}(l-r)$.

The ground state of the combined system can be described by the single Slater determinant

$\Psi_{0}=\frac{1}{4} 1(L+R)^{\alpha}(L+R)^{\beta}(L-R)^{\alpha}(L-R)^{\beta} \mid$.

When we make use of the well-known determinant properties for linear combinations of columns, we find that $\Psi_{0}=\left|L^{\alpha} L^{\beta} R^{\alpha} R^{\beta}\right|$.

We consider now an excited triplet state of the combined system. We promote one electron from an orbital $2^{-1 / 2}(L-R)$ to an orbital $2^{-1 / 2}(l-r)$ so that we get a one determinantal wavefunction for a triplet state

$\Psi_{1}=\frac{1}{4}\left[(L+R)^{\alpha}(L+R)^{\beta}(L-R)^{\alpha}(l-r)^{\alpha} \mid\right.$.

The spatial symmetry with respect to the symmetry plane is left unchanged by this excitation, hence it is symmetric, as it is for the ground state. We could have chosen an excitation that changes the symmetry; this leads to results similar to the ones presented here and will not be considered any further.

Using some well-known determinant properties, one finds for $\Psi_{1}$ the equivalent expression

$$
\begin{gathered}
\Psi_{1}=\frac{1}{2}\left|L^{\alpha} L^{\beta} l^{\alpha} R^{\alpha}\right|+\frac{1}{2}\left|R^{\alpha} R^{\beta} r^{\alpha} L^{\alpha}\right| \\
\quad+\frac{1}{2}\left|L^{\alpha} L^{\beta} R^{\alpha} r^{\alpha}\right|+\frac{1}{2}\left|R^{\alpha} R^{\beta} L^{\alpha} l^{\alpha}\right| .
\end{gathered}
$$

The first two terms are of a charge transfer type $[19,20]$, in which one has three electrons in one molecule and one electron in the other one. It is clear that this wavefunction alone cannot give an adequate description of a low lying excited state of two uncoupled molecules. There is, however, another wavefunction $\Psi_{2}$ with the same spatial symmetry and with the same energy as $\Psi_{1}$, which is obtained by promoting one electron from an orbital $2^{-1 / 2}(L+R)$ to $2^{-1 / 2}(l+r)$, so that the wavefunction becomes:

$\Psi_{2}=\frac{1}{4}\left|(L-R)^{\alpha}(L-R)^{\beta}(L+R)^{\alpha}(l+r)^{\alpha}\right|$.

This function can be rewritten as:

$\Psi_{2}=-\frac{1}{2}\left|L^{\alpha} L^{\beta} l^{\alpha} R^{\alpha}\right|-\frac{1}{2}\left|R^{\alpha} R^{\beta} r^{\alpha} L^{\alpha}\right|$

$$
+\frac{1}{2}\left|L^{\alpha} L^{\beta} R^{\alpha} r^{\alpha}\right|+\frac{1}{2}\left|R^{\alpha} R^{\beta} L^{\alpha} l^{\alpha}\right| \text {. }
$$

We see that also for $\Psi_{2}$ the first two terms are of a charge transfer type, and hence $\Psi_{2}$ cannot be a satisfactory function either. For uncoupled systems the lowest triplet state of the combined system is neither $\Psi_{1}$ nor $\Psi_{2}$ but $2^{-1 / 2}\left(\Psi_{1}+\Psi_{2}\right)$. We see this as follows: for the matrix element $\left\langle\Psi_{1} H \Psi_{2}\right\rangle$ we find with the usual methods:

$$
\begin{aligned}
& \left\langle\Psi_{1} H \Psi_{2}\right\rangle=-\frac{1}{4} \int\{L(1)+R(1)\} *\{L(1)-R(1)\} \\
& \times\left(e^{2} / r_{12}\right)\{l(2)-r(2)\} *\{l(2)+r(2)\} \mathrm{d} r_{12} \\
& =-\frac{1}{4}\left\{J_{L I}-J_{L r}-J_{R 1}+J_{R r}\right\},
\end{aligned}
$$

in which $J_{u}$ etc. are Coulomb integrals. Because of the symmetry of the system we have $J_{L I}=J_{R r}$ and $J_{R l}=J_{L r}$ so that $\left(\Psi_{1} H \Psi_{2}\right\rangle=\frac{1}{2}\left(J_{L r}-J_{L l}\right)$. The

Coulomb integral $J_{L l}$ is positive; for two uncoupled molecules the integral $J_{L r}$ will certainly be smaller than $J_{L}$; for two molecules at infinite distance $J_{L r}$ is zero, hence for uncoupled molecules $\left(\Psi_{1} H \Psi_{2}\right)$ is always negative. The secular equations for this simple problem lead to a lowest triplet state function:

$$
\begin{aligned}
\Psi_{\mathrm{S}} & =2^{-1 / 2}\left(\Psi_{1}+\Psi_{2}\right) \\
& =2^{-1 / 2}\left\{\left|L^{\alpha} L^{\beta} R^{\alpha} r^{\alpha}\right|+\left|R^{\alpha} R^{\beta} L^{\alpha} I^{\alpha}\right|\right\},
\end{aligned}
$$

when $\left\langle\Psi_{1} H \Psi_{2}\right\rangle<0$. We see that the charge transfer terms in $\Psi_{1}$ and $\Psi_{2}$ cancelled each other. The two terms which are left make the probability to find the excitation on either the right hand side or the left hand side both equal to one half and the two unpaired spins are either both in the right hand ring or both in the left hand ring of the system with equal probability.

The foregoing considerations apply also to the molecules used in our experiments. When the bridges are long enough so that the aromatic systems hardly overlap, the system as a whole will behave like weakly coupled aromatic systems. In particular, the lower triplet states will be such that both unpaired spins are simultaneously in one ring system or simultaneously in the other and the spin distribution and related properties such as $D$ and $E$ values will be close to those of a single aromatic ring system with aliphatic substitution. The spin density is clearly spread over the two rings and gives each ring half the density one finds for one isolated molecule in the triplet state.

When the two aromatic systems are sufficiently close together to allow appreciable overlap between the two ring systems, we get a different situation. In the first 
place, the integral $\left(\Psi_{1} H \Psi_{2}\right)$ becomes less negative because for smaller values of the distance between the ring systems $J_{L}$ is no longer zero but can get an appreciable positive value. In the second place the orbitals $2^{-1 / 2}(I,+R)$ and $2^{-1 / 2}(L-R)$ etc. are no longer recessarily degenerate and consequently $E_{1} \neq E_{2}$ when $\left|E_{1}-E_{2}\right|<\left\langle\Psi_{1} H \Psi_{2}\right\rangle$ we still get an appreciable mixing of $\Psi_{1}$ and $\Psi_{2}$ and the system will still behave approximately as two weakly coupled aromatic systems. If, however, $\left.\left|E_{1}-E_{2}\right|\right\rangle\left\langle\Psi_{1} H \Psi_{2}\right\rangle$ one must expect the functions $\Psi_{1}$ and $\Psi_{2}$ to give adequate description of excited states rather than their line.ur combinations and the spin distribution will be more spread over the entire molecule, in particular it is no longer necessary that both unpaired spins are certainly simultaneously in the same aromatic ring. Under those conditions it is no longer to be expected that the $D$ and $E$ values resemble those of the separate ring systems.

When the two ring systems of a phane are different and in particular when one of the two is a donor, the other an acceptor, one expects that a charge transfer state will be the lowest triplet state and hence one will have an unpaired electron in each of the subsystems. In case the two unpaired electrons are in two different ring systems, their distances will usually be larger than when they are in one and the same ring. As the $D$ value depends essentially on $\left\langle r^{-3}\right\rangle$ one expects the $D$ val. ues for charge transfer phanes to be smaller than those for symmetrical pirianes or single molecules.

Other authors have also considered the neutral excitation states and the charge transfer states $[19,20]$ in a study of the excimer fluorescence of singlet states of paracyclophane and molecular complexes [26]. In the work quoted above, the two states are postulated separately and then considered together in a configuration interaction calculation. What we wanted to show here is that both types of functions appear very naturally in a molecular orbital description which is adapted to double molecules. We have done this for excited triplet states. However, one derives easily wave functions for singlet excited states similar to the ones given above for triplet states.

There is, however, a reason to be very careful with the assumption that the behaviour of excited singlet states and triplet states is very similar. It has been customary to assume that the orbitals in a singlet state and a triplet state are identical or almost identical.

This latter assumption appears to be highly question- able. Very detailed considerations for many atoms [27-30] and for excited states of $\mathrm{H}_{2}[28,31]$, $\mathrm{C}_{2} \mathrm{H}_{4}$ [32], $\mathrm{C}_{2} \mathrm{H}_{2}$ and $\mathrm{CO}_{2}$ [33] and trans-butadiene [34] show that the wavefunction for an excited singlet state is much more diffuse than the wavefunction for the corresponding triplet state, which is rather contracted towards the nuclei. In.an SCF approach it appears to be the outer orbital which is vastly different in singlet and triplet excited states [29,30,32].

The inequality formalism for energy differences as derived in ref. [29] strongly suggests this difference in orbital behaviour for singlets and triplets. The formalism in ref. [29] is general although it has been applied to atomic energy differences so far [30]. All available evidence suggests that the contraction of an outer triplet orbital and the expansion or diffuseness of an outer singlet orbital is a general feature for atoms and molecules.

When this is true, the rather diffuse $\pi^{*}$ singlet orbitals of the two halves of a phane may overlap already considerably at a distance between the subsystems at which the triplet orbitals behave still like almost separate orbitals.

Due to the difference in size of the $\pi^{*}$ orbital in singlet and triplet excited states one may expect also that for guest molecules in a crystal or a glass, the coupling between an excited singlet state and the lattice could be stronger than the coupling between a triplet state and lattice.

\section{Experimental}

The preparation and purification of the phanes was described before $[14-16,25]$. Two types of samples were prepared: samples of phanes in polymethylmethacrylate and single crystals of fluorene doped with phanes. For the plastic samples a solution of $10^{-2}$ $\mathrm{mol} / \mathrm{l}$ of the phane in freshly destilled methylmethacrylate $(2.5 \mathrm{ml})$ and dithio-bis-formic acid $o, 0$-diisopropylester $(500 \gamma)$ as initiator was made. This solution was transferred into a glass sample tube with a diameter of $5.5 \mathrm{~mm}$. At least four freeze-thaw cycles were carried out before sealing under high vacuum. The sealed tubes were polymerized by irradiating with light of a $150 \mathrm{~W}$ lamp for about five hours. After tempering the tubes at $50^{\circ} \mathrm{C}$ for at least 24 hours, small rods of $3 \mathrm{~mm}$ and $5 \mathrm{~mm}$ diameter and about $10 \mathrm{~mm}$ length were pre- 
pared and polished. The fluorescence and phosphorescence spectra were taken directly after the preparation of the samples. The same procedure was carried out with the corresponding monomers as reference samples.

$[2.2](2,7)$ phenanthrenophane and $[2.2](1,4)$ naphthalenophane were also measured in low concentration ( $<1000 \mathrm{ppm}$ ) in fluorene crystals. The fluorene was synthesized in order to obtain it free from impurities, in particular anthracene. This procedure reduces the concentration of impurities below $1 \mathrm{ppm}$, and the high resolution phosphorescence spectra at $4.2 \mathrm{~K}$ of a pure fluorene crystal was the same as shown by Bree and Zwarich [35].

For the fluorescence and phosphorescence spectra a spectrometer Fluorispec model SF-100 (BairdAtomic) with a cryostat for liquid helium measurements was used. The spectra shown are uncorrected with respect to the intensity of the lamp and to the sensitivity of the photomultiplier. The resolution of this spectrometer is $1.5 \mathrm{~nm}$, which was found to be sufficient for the broad emission spectra of the phanes. All samples were measured at room temperature, $78 \mathrm{~K}$ and $4.2 \mathrm{~K}$. Some spectra were taken at $1.9 \mathrm{~K}$ as well, but no appreciable changes could be observed as compared to $4.2 \mathrm{~K}$.

A high sensitive X-band ESR spectrometer [36] with liquid helium cryostat was used for measuring $D$ and $E$ values of the phanes. Because of the weakness of the ESR signals the relative errors are large, and from some samples no signals could be obtained. For that reason, the zero field splitting parameters $D$ and $E$ were determined by optical detection of magnetic resonance (ODMR) in zero field. At $1.5 \mathrm{~K}$ the method used was the analysis of phosphorescence transients after a saturating fast passage $[37,38]$. The ODMR apparatus was in principle the same as described by Zuclich et al. [39]. Beside the zero field splitting parameters also the relative steady state populations and radiative rate constants were obtained.

\section{Results}

In fig. 1 the [2.2]phanes studied as well as the corresponding monomers are shown. In the case of [2.2]$(2,7)$ phenanthrenophane the sample was a $5: 1$ mixture of the species 13 and 14 [15].

Figs. 2-7 show the fluorescence and phosphorescence

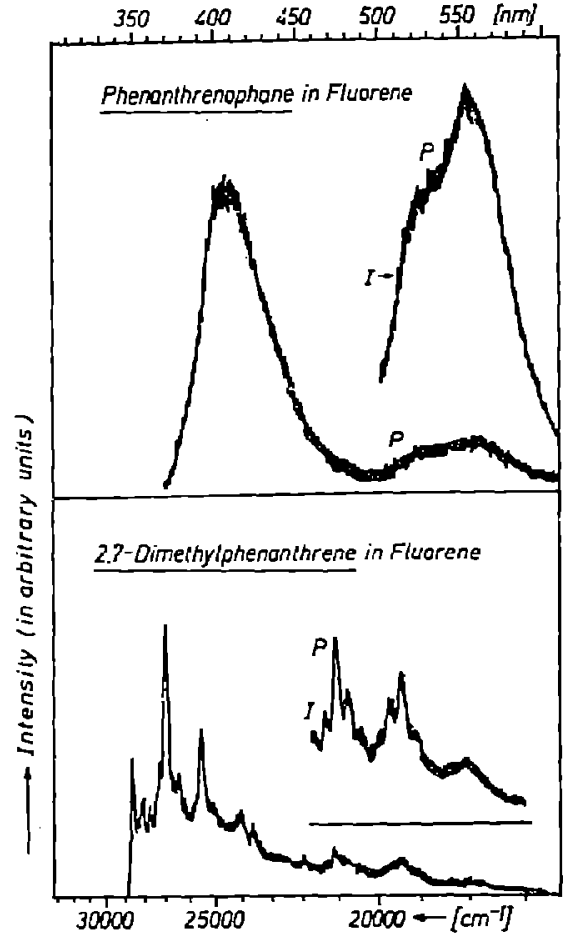

Fig. 2. Fuorescence and phosphorescence (P) spectra of 2,7dimethylphenanthrene (12) and $[2.2](2,7)$ phenanthrenophane $(13,14)$ in a fluorene host cry stal at $4.2 \mathrm{~K}$ (see also section 4).

(P) emission spectra of several phanes and in some cases of the corresponding monomers at $4.2 \mathrm{~K}$. Fig. 2 shows the fluorescence and phosphorescence (P) spectra of 2,7-dimethylphenanthrene (12, lower part) and $[2.2](2,7)$ phenanthrenophane (13/14, upper part) in a fluorene host crystal. The phosphorescence part (P) of $[2.2](2,7)$ phenanthrenophane was also observed with higher exciting light level intensity ( $\mathrm{I}$ in fig. 2). In the case of 2,7-dimethylphenanthrene the exciting wavelength was $340 \mathrm{~nm}(1.5 \mathrm{~nm}$ half width), in the case of $[2.2](2,7)$ phenanthrenophane $365 \mathrm{~nm}(1.5 \mathrm{~nm}$ half width and $6 \mathrm{rm}$ half width for I). Both exciting energies are far below the $S_{0} \rightarrow S_{1}$ transition of the fluorene host system. For comparison, figs. 3 and 5 show the fluorescence and phosphorescence (P) emission of phenanthrene (I1, lower part, fig. 3), 2, 7dimethylphenanthrene (12, lower part, fig. 5) and $[2.2](2,7)$ phenanthrenophane $(13,14$, fig. 3$)$ in a polymethylmethacrylate (PMMA) matrix. The exciting 


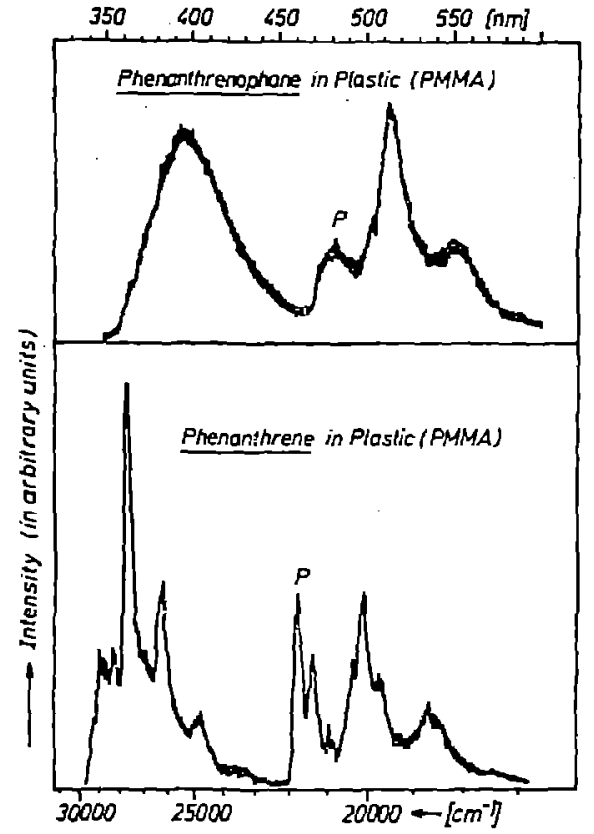

Fig. 3. Fluorescence and phosphorescence (P) spectra of phenanthrese (11) and $[2.2](2,7)$ phenanthrenophane $(13,14)$ in polymethylmethacrylate (AMMA) at $4.2 \mathrm{~K}$.

wavelength hare was $320 \mathrm{~nm}$ ( $21 \mathrm{~nm}$ half width in both cases).

Fig. 4 shows the sinilar emission spectra of $[2.2]\left(4,4^{\prime}\right)$ biphenylophane $(8)$ and of $5,6,17,18$. tetrahydro[2.2] $(2,7)$ phenanthrenophane $(10)$ in PMMs (exciting wavelength $320 \mathrm{~nm}, 21 \mathrm{~nm}$ half width). The emission spectra of 19,20 -dihydro 2$]\left(4,4^{\prime}\right)$ biphenylo [2] $(2,7)$ phenanthrenophane (9) in PMMA is not shown because it is rearly identical with the spectra of 8 and 10 (fig. 4). This is not surprising since all three molecules possess the same $\pi$-electron orbital system.

The emission spectra of biphenyl (6) and 9,10-dihydrophenanthrene (7) in PMMA could not be obtained because the $S_{1}$ and $T_{1}$ states lie above the corresponding states of PMMA [40].

Fig. 5 shows the fluorescence and phosphorescence (P) emission of 2,7-dimetliylphenanthrene (12) in PMMA and on top the emission of $[2]\left(4,4^{\prime}\right)$ biphenylo [2] $(2,7)$ phenanthrenophane (15) in PMMA under the same conditions as in fig. 4 .

The last two figures, 6 and 7 , show the fluorescence and phosphorescence (P) emission of naphthalene (1)

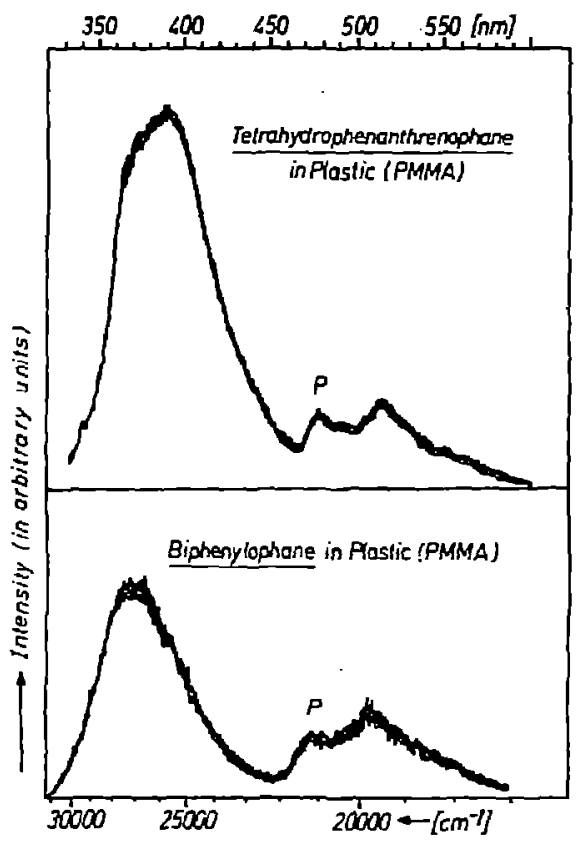

Fig. 4. Fluorescence and phosphorescence (P) specta of [2.2] $\left(4,4^{\prime}\right)$ biphenylophane (8, on top) and of $5,6,17,18$ tetrahydro $[2.2](2,7)$ phenanthrenophane $(10)$ in PMMA at $4.2 \mathrm{~K}$.

in PMMA (lowest part, fig. 6), 1,4-dimethylnaphthalene in PMMA (middle, fig. 6), 2,6-dimethylnaphthalene (2) in PMMA (lower part, fig. 7) as well as anti-[2.2]$(1,4)$ napthalenophane $(4)$ in PMMA (fig. 6, upper part) and $[2.2](2,6)$ naphthalenophane (5) in PMMA (fig. 7, upper part) (exciting wavelength for 1 and 2: $290 \mathrm{~nm}$ with $21 \mathrm{~nm}$ half width, for 4 and 5: $330 \mathrm{~nm}$ with 21 $\mathrm{nm}$ half width). In the case of naphthalene (1), 1,4 dimethylnaphthalene and 2,6-dimethylnaphthalene (2) the phosphorescence part is also shown with a resolution of $6 \mathrm{~nm}$ (I in figs. 6 and 7), whereas in the case of anti-[2.2] $(1,4)$ naphthalenophane (4) and [2.2]$(2,6)$ naphthalenophane $(5)$ the very weak phosphorescence emission (P) was taken with a rotor and a resolution of only $21 \mathrm{~nm}$ (II in figs. 6 and 7). The spectrum of anti-[2.2] $(1,4)$ naphthalenophane in a fluorene host crystal is not shown because it looks identical to that shown in fig. 6 in PMMA.

In table 1 the zero field splitting parameters $D$ and $E$ are given of the phanes investigated in PMMA and of the corresponding monomers in PMMA as well as in some cases in host crystals. The $D$ and $E$ values of $[2.2](2,7)$ phenanthrenophane in a fluorene host 


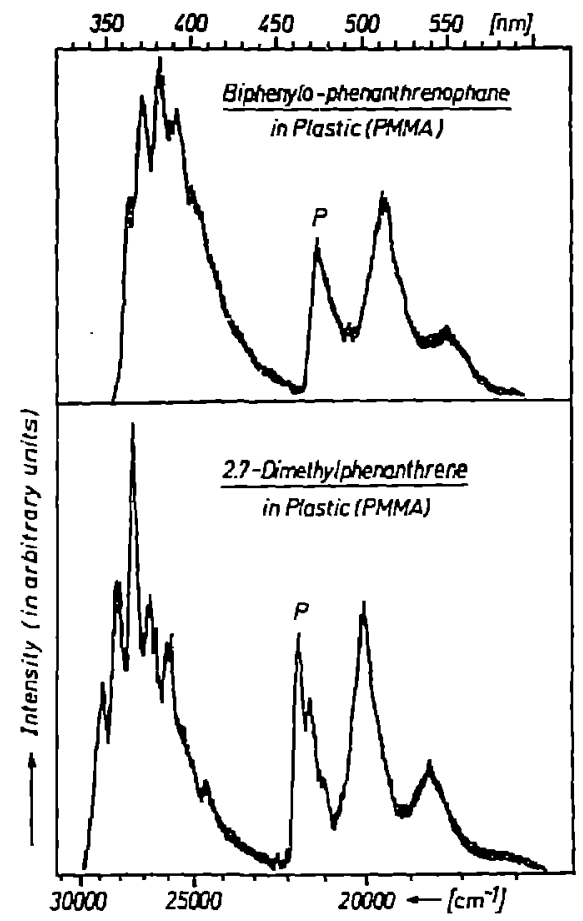

Fig. 5. Fluorescencc and phosphorescence (P) spectra of 2,7dimethylphenanthrene (12) ard of [2] (4.4')biphenylo [2]$(2,7)$ phenanthrenophane $(15)$ in PMMA at $4.2 \mathrm{~K}$.

crystal are also given in table 1 . The zero field splitting parameters were obtained by optically detected magnetic resonance (ODMR) technique and partly by normal ESR methods. The error in the case of the ESR method is about $5 \%$, while in the case of the ODMR technique the error is much smaller and mostly caused by the uncertainty due to the linewidth of the micro. wave transitions (typical about $\pm 0.0002 \mathrm{~cm}^{-1}$ ).

Table 2 shows the relative radiative rate constants and steady state populations of naphthalene (1), 1,4dimethylnaphthalene, 2,6-dimethylnaphthalene (2), anti-[2.2] $(1,4)$ naphthalenophane (4) and $[2.2](2,6)$ naphthalenophane (5) in PMMA.

\section{Discussion}

\subsection{Biphenylo- and phenanthrenophanes}

The main striking difference between the emission spectra of the phanes as compared to the monomers

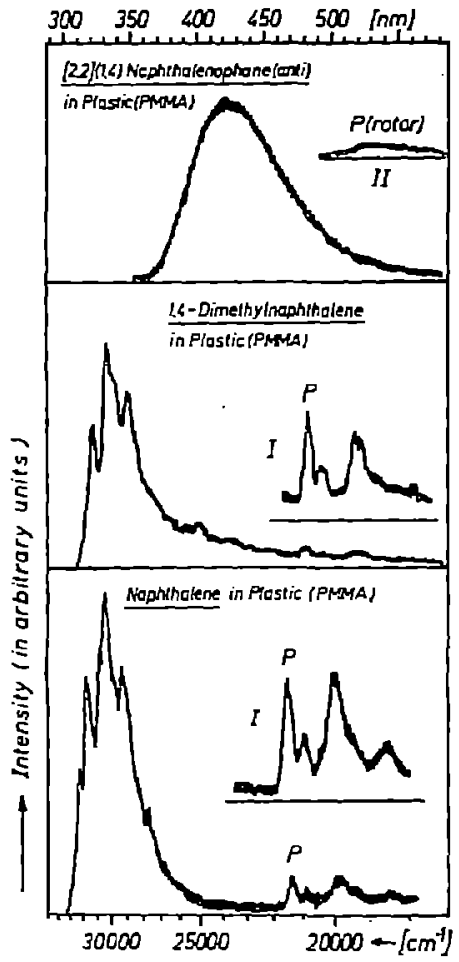

Fig. 6. Fluorescence and phosphorescence (P) spectra of naphthalene (1), 1,4-dimethylnaphthalene and anti-[2.2]$(1,4)$ naphthalenophane $(4)$ in PMMA at $4.2 \mathrm{~K}$.

is that the phanes exhibit broad structureless emission bands shifted to the red which are typical of dimers or excimers, while the spectra of the corresponding monomers show sharp vibrational structure. This is particularly true for phanes which consist of two identical halves, such as $[2.2](2,7)$ phenanthrenophane (13/14, figs. 2 and 3), 5,6,17,18-tetrahydro[2.2] $(2,7)$ phenanthrenophane (10), and $[2.2]\left(4,4^{\prime}\right)$ biphenylophane (8, fig. 4). The 19,20-dihydro[2] $\left(4,4^{\prime}\right)$ biphenylo[2] $(2,7)$ phenanthrenophane $(9)$ belongs to this group as well since the two units, al though not identical, possess the same $\pi$-electron system which is relevant for the properties discussed here. [2] $\left(4,4^{\prime}\right)$ biphenylo$[2](2,7)$ phenanthrenophane (15, fig. 5), a phane with two different aromatic units, on the other hand shows as compared with dimethylphenanthrene a somewhat broadened spectrum in which, however, a vibrational structure is still discernible.

This general behaviour can be well understood in 


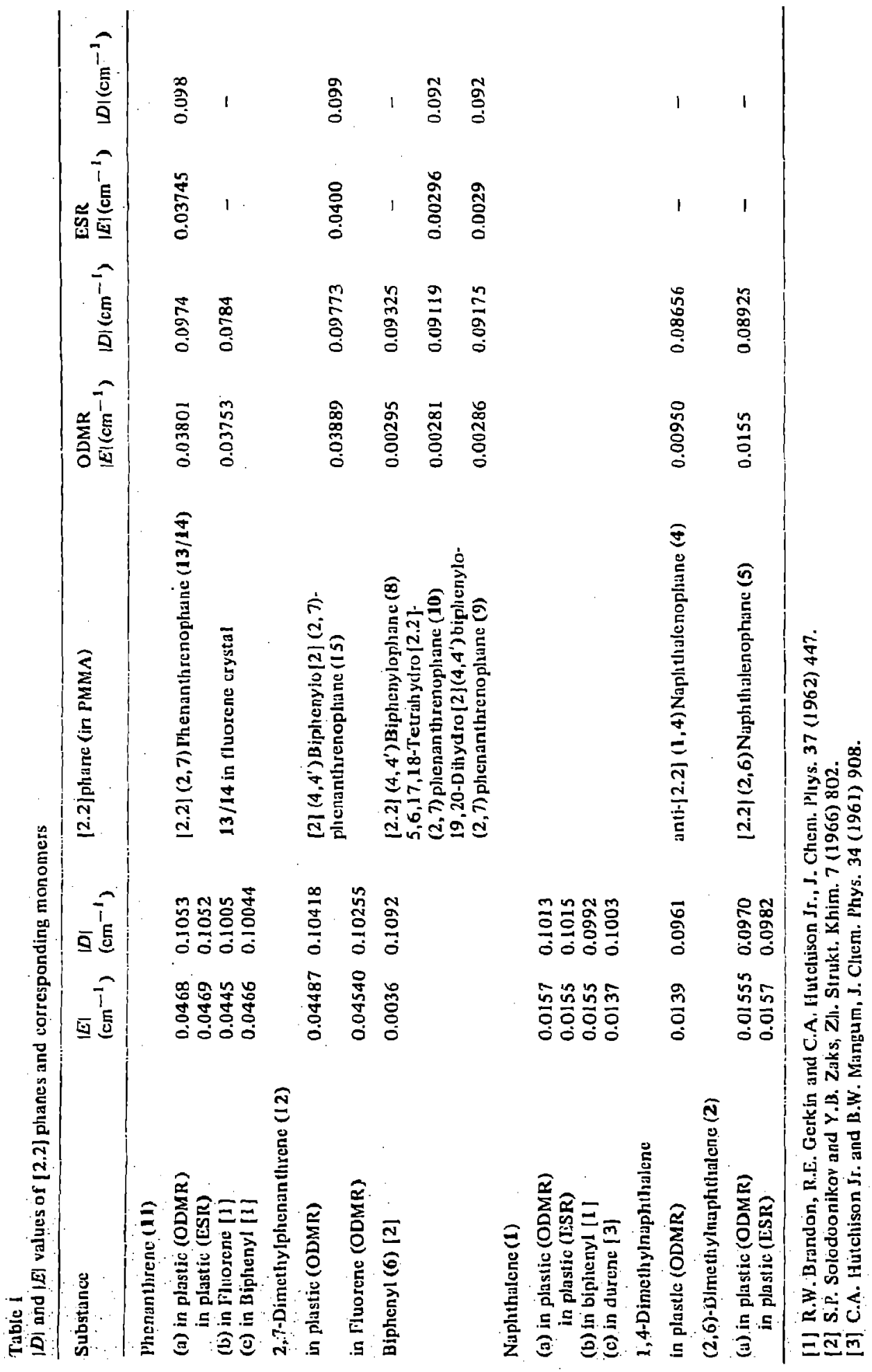


Taule 2

Relative radiative rate constants and steady state populations of naphthalenes and naphthalenophanes ${ }^{3)}$

\begin{tabular}{|c|c|c|}
\hline Naphthalene & $k_{x}^{\Gamma} \neq 0, k_{y}^{5}=k_{z}^{r}=0$ & $n_{z}>n_{y}>n_{x}$ \\
\hline 1,4-Dimcthylnaphthalene & $k_{x}^{\mathrm{r}} \neq 0, k_{y}^{\mathrm{r}}=k_{z}^{\mathrm{I}}=0$ & $n_{z}>n_{y}>n_{x}$ \\
\hline 2,6-Dimethylnaphthalene & $k_{z}^{5} \neq 0, k_{y}^{5}=k_{x}^{5}=0$ & $n_{x}>x_{y}>n_{z}$ \\
\hline anti-[2.2](1,4) Naphthalenophane & $k_{x}^{5}>k_{z}^{5} \neq 0, k_{y}^{5}=0$ & $n_{z}>n_{y}>n_{x}$ \\
\hline$[2.2](2,6)$ Naphthalenophane & $k_{z}^{\mathrm{r}}>k_{x}^{\mathrm{r}} \div 0, k_{y}^{\mathrm{r}}=0$ & $n_{x}>n_{y}>n_{z}$ \\
\hline
\end{tabular}

a) Here we have defined: triplet $x$-sublevel is top level, $y$-sublevel middle level and a $2|E|$ transition means an $x \rightarrow y$ transition, $k_{i}^{\Gamma}$ radiative rate constant and $n_{i}$ steady state population of the ith triplet sublevel $(i=x, y, z)$.

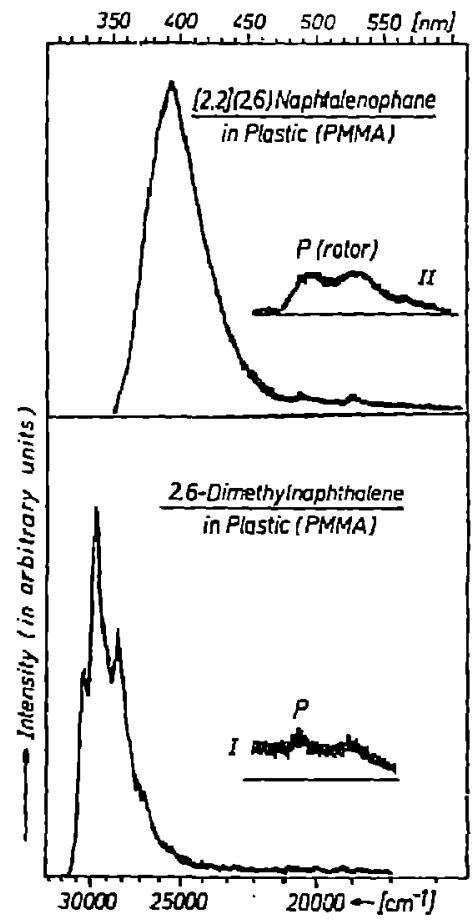

Fig. 7. Fluorescence and phosphorescence (P) of 2,6-dimethylnaphthalene (2) and of $[2,2](2,6)$ naphthalenophane $(5)$ in PMMA at $4.2 \mathrm{~K}$.

terms of the theory given above. The $\left(\mathrm{CH}_{2}-\mathrm{CH}_{2}\right)$ bridges are so short that they force the two units closely together with the result that one observes the typical emission spectra of dimers. The case of the phane 15 is somewhat different. The energies of the first excited singlet and triplet state $S_{1}$ and $T_{1}$ of the biphenyl are higher than those of the phenanthrene. Hence the phenanthrene acts as a trap for the energy in the phane system; in other words, the emission from the phane $\mathbf{1 5}$ is essentially an emission from a phenanthrene molecule which is somewhat perturbed by the interaction with an adjacent biphenyl.

In addition to this general behaviour, a detailed comparison of the spectra provides additional information. While the fluorescence spectra of 8,10 and 13/14 in PMMA show only one broad structureless band, the phosphorescence exhibits still some structure, which is most marked in the case of $13 / 14$. This behaviour supports the idea discussed in part Il that the wavefunction of an excited singlel state $S_{1}$ is more diffuse than the wavefunction of the corresponding triplet state $T_{1}$ and hence at a given distance the overlap between the two halves of the phane is higher in the singlet state than in the triplet state.

An unexpected effect was observed when comparing the emission spectra of 2,7-dimethylphenanthrene and 13/14 in a fluorene single crystal (fig. 2) and in PMMA (figs. 3 and 5). The spectra of the monomer are very similar with the only difference that the vibrational structure is somewhat better resolved in fluorene as compared to the solid solution in PMMA. In contrast, both the fluorescence and phosphorescence of $13 / 14$ are considerably more shifted to the red (about $3000 \mathrm{~cm}^{-1}$ ) in the fluorene matrix than in PMMA and the structure of the phosphorescence, a clearly resolved triplet in PMMA, has disappeared in the fluorene crystal except for a weak shoulder on the high energy side.

Parallel to these optical results the $D$ value of the excited triplet state of $13 / 14$ is found to be about $20 \%$ smaller in the fluorene crystal than in PMMA (see table 1). Since we do not see any reason why the intramolecular trarisannular interaction within the phane should be stronger in the fluorene crystal than in 
PMMA, this result seems to indicate that the phane as a whole has a stronger interaction with the neighbouring fluorene molecules than with the PMMA.

When discussing the $D$ values we should keep in mind that they are very similar for most aromatic molecules and less sensitive to small interactions with the surroundings, while the $E$ values can differ by orders of magnitude and are very sensitive to a change in the symmetry. The general result of our measurement is that the $D$ and $E$ values of the phanes are all below the values of the respective nonomers. In good agreement with the emission spectra we find a comparatively small reduction of the $D$ value in PMMA in the [2] $\left(4,4^{\prime}\right)$ biphenylo[2] $(2,7)$ phenanthrenophane (15) of about $7 \%$ as compared to the monomer 2,7 . dimethylphenanthrene indicating a somewhat perturbed triplet state of phenanthrene; a microwave transition corresponding to the zero field splitting parameters of biphenyl could not be detected. All three phanes with the $\pi$-electron system of biphenyl exhibit in PMMA a larger reduction of the $D$ value of about $17 \%$ with respect to biphenyl monomer and also a reduction of the $E$ value which is already very small for biphenjl itself. These results are in at least qualitative agreement with wavefunctions discussed in part II which permit the electrons to be on the average a little farther apart from each other in the phane than in the monomer with the result of a somewhat smaller dipolar coupling.

The rather small reduction in the $D$ values indicates that the contribution of the charge transfer states for the wavefunction of the triplet states of the phanes is small in the case of the phanes with two identical halves discussed here. This is in striking contrast to the $D$ values obtained with another type of phanes consisting of two units between which a charge transfer is known to occur [41]; the $D$ values of such phanes are found to be smaller by a factor between two and six as compared to the corresponding monomers.

A special case is again 13/14 in PMMA and in a fluorene crystal. While the reduction of the $D$ value of about $7 \%$ is comparatively small in PMMA, the $D$ value measured in fluorene is $0.078 \mathrm{~cm}^{-1}$, the smallest $D$ value measured for any of the phanes which we have investigated in this work; it corresponds to a reduction of more than $20 \%$ as compared to the 2,7-dimethylphenanthrene in the same matrix.

\subsection{Naphthalenophanes}

Both naphthalenophanes investigated show the broad structureless fluorescence typical of dimers. The fluorescence band of anti-[2.2] $(1,4)$ napthalenophane (4) in PMMA (fig. 6) is broader and farther shifted to the red than the one of $[2.2](2,6)$ naphthalenophane ( 5 , fig. 7 ) indicating a stronger interaction between the two halves in the former. In accordance with the geometrical structure a still further red-shift was reported for the syn-[2.2] $(1,4)$ naphthalenophane (3) in an EPA matrix (maximum intensity at $460 \mathrm{~nm}$ ) [23]. The fluorescence spectrum of 4 in PMMA (maximum intensity at $420 \mathrm{~nm}$ ) shows a somewhat larger rea. shift than the spectrum in an EPA matrix [23], which is probably due to a solvent effect. Another indication for the weaker interaction of the $\pi$-electrons in [2.2]$(2,6)$ naphthalenophane $(5)$ as compared to anti-[2.2]$(1,4)$ naphthalenophane $(4)$ is the narrower width of the fluorescence band which corresponds to a weaker electron-phonon coupling strength [42]. The larger overlap in 4 as compared to 5 is not surprising and can be understood on a geometrical bases. We knew from $\mathrm{X}$-ray studies that the aromatic rings of the phanes deviate considerably from planarity because of strain in the phane system and transannular repulsion of the $\pi$-electrons. Due to the energetically more favourable possibility of distributing the defomation upon more C...C bonds in 5 than in 4 , the average transannular distance in $\mathbf{5}$ is longer. Therefore, the overlap in $\mathbf{5}$ is weaker than in 4.

This fact is also shown by some features of the triplet state: while the reduction of the $D$ value (table 1) is $10 \%$ for 4 in comparison to 1,4 dimethylnaphthalene, the $D$ value of 5 is reduced by $7 \%$ as compared to 2,6-dimethylnaphthalene (2). Further, if we compare the phosphorescence of $[2.2](2,6)$. naphthalenophane (5) and of 2,6-dimethylnaphthalene (2) in fig. 7, we find no red-shift for the phane; the two weak emission peaks occur at the same wavelength as in 2,6-dimethylnaphthalene, in contrast to the phosphorescence of anti-[2.2] $(1,4)$ naphthalenophane (4) which shows a red-shift with respect to 1,4-dimethylnaphthalene.

Additional information on the properties of the excited triplet states can be obtained by studying intersystem crossing rates, steady state populations, relaxation rates, and radiative and non-radiative decay 
constants of the phanes as compared to the corresponding monomers.

A typical feature of the phosphorescence of the naphthalenophanes is its weak intensity which is, for instance, for anti- $[2,2](1,4)$ naph thalenophane (4) about one order of magnitude weaker than for 1,4 dimethylnaphthalene. We see from table 2 that in 1,4 dimethylnaphthalene only the top $x$-level is radiative, while in the phane 4 the $x$ and $z$ sublevel radiates. The total rate constant $k_{x}=1.6 \mathrm{~s}^{-1}$ of the monomer is drastically enhanced in the phane 4 where $k_{x}=15.3 \mathrm{~s}^{-1}$ and $k_{z}=1.7 \mathrm{~s}^{-1}$, while $k_{y}$ is only about $0.2 \mathrm{~s}^{-1}$. These results show that the weaker intensity of the phosphorescence is due to an increase of the nonradiative decay of the triplet state.

When measuring the decay constants $k_{i}$, however, one must take the spin lattice relaxation into account as well. At $1.5 \mathrm{~K}$ the apparent decay constant of the phosphorescence seems to be of the order of $0.2 \mathrm{~s}^{-1}$. This observation is due to the slow spin lattice relaxation between the radiationless $y$ sublevel and the radiative $x$ and $z$ sublevels (definitions see table 2). Assuming fast spin lattice relaxation at higher temperatures results in a total rate constant $k=5.68 \mathrm{~s}^{-1}$ corresponding to a phosphorescence life-time of $0.176 \mathrm{~s}$ which is in very good agreement with the one of $0.18 \mathrm{~s}$ measured by Froines and Hagermann [23].

In the $[2.2](2,6)$ naphthalenophane (5) the highest rate constant is also enhanced to $k_{z}=7.5 \mathrm{~s}^{-1}$ as compared to the value $1.6 \mathrm{~s}^{-1}$ for the 2,6-dimethylnaphthalene; both the $x$ and $z$ sublevels become radiative in contrast to the monomer. This shows as well as the result obtained with $[2.2](1,4)$ naphthalenophane (4) how sensitive the rate constants are to interactions within the $\pi$-electron system.

Another effect which requires further investigation is the linewidth of the microwave transitions. The linewidth of naphthalene in zero field in a fluorene crystal is of the order of $1-2 \mathrm{MHz}$ and in PMMA about $20 \mathrm{MHz}$ due to the inhomogeneity broadening because of the random orientation. The linewidths of the naphthalenophanes on the other hand in PMMA reach values up to $100 \mathrm{MHz}$. Similar linewidths were observed for the other phanes as well with the exception of $[2.2](2,7)$. phenanthrenophane $(13,14)$ in a fluorene crystal which behaves differently also in this respect; its linewidth was found to be about 2-10 MHz.

\section{Conclusions}

The experimentally observed fluorescence spectra of a number of symmetric [2.2] phanes show the typical behaviour of emission spectra of dimers and excimers, i.e. in the excited singlet state the electronic orbitals of the two halves of the system are closely coupled.

Three properties of the excited triplet state $T_{1}$ of the phanes were measured:

1. The zero field splitting parameters $D$ and $E$,

2. phosphorescence emission spectra,

3. decay constants of the three electronic sublevels.

1. The zero field splitting parameters $D$ and $E$ which depend on the dipolar coupling between the two unpaired electrons were always found to be somewhat smaller than those of the monomers, but the difference is not large, typical of the order of $10 \%$. This shows that the two unpaired electrons have a high probability to be at a given time in the same half of the molecule because otherwise we would expect much lower $D$ values.

Although the two unpaired electrons are localized at a given time with high probability in one half, the two electron spin density îs evenly distributed over the entire system which means, classically speaking, that the two unpaired electronic spins spend half of the time in one half of the system and the other half of the time in the other half. The net result is a time average of a one electron spin density per half which determines the hyperfine coupling.

2. The phosphorescence emission spectra show, in contrast to the fluorescence, some remaining structure indicating a somewhat weaker coupling between the two halves. This behaviour can be understood on the basis of previous work $[28,31-34]$ with smaller systems which shows that the spatial extension of the triplet orbital is considerably smaller than the one of the singlet orbital and hence, at a given distance which is determined by the $\left(\mathrm{CH}_{2}-\mathrm{CH}_{2}\right)$ bridges, the interaction among the triplet orbitals is smaller than the interaction among the singlet orbitals.

3. The decay rate constants $k_{i}$ of the phanes are in most cases much shorter than those of the corresponding monomers indicating again a strong interaction among the two halves of the system.

A special type of substances are the phanes with two different aromatic units; the more the electronic 
orbitals and the energies of the excited states of the two monomers differ from each other the more the properties of the corresponding phane become similar to those of the monomer with the lower excited state with some perturbation by the other part. A typical example of this type of phanes is the biphenylophenanthrenophane (15) which exhibits both fluorescence and a phosphorescence emission spectra with fairly well resolved structure which resembles quite well the spectra of the monomer dimethylphenanthrene.

These results can be summarized by saying that the answer to the question whether the two halves of the phanes are very strongly or somewhat more weakly coupled depends on the physical properties which one takes as a criterium.

\section{Acknowledgement}

We thank Mr. H. Zimmernann for synthesizing the fluorene material and preparing the crystals, as well as him and Miss Ch. Lobensteiner for preparing the PMMA samples.

\section{References}

[1] T. Förster and K. Kasper, Z. Physik Chem. (Frankfurt) I (1954) 19.

[2] T. Förster and K. Kasper, 2. Elektrochem. 59 (1955) 976.

[3] B. Stevens, Nature 192 (1961) 725.

[4] T. Förster, C.O. Leiber, H.P. Seidel and A. Weller, Z. Phys. Chem. NF 39 (1963) 265.

[5] J.B. Birks and L.G. Christophorou, Spectrochim. Acta 19 (1963) 401.

[6] F.J. Smith, A.T. Armstrong and S.P. MoGlynn, J. Chem: Fliys. 44 (1966) 442.

[7] J.B. Birks, D.J. Dyson and I.H. Munro, Proc. Roy. Soc. A 275 (1963) 575.

[8] J.B. Birks and J.B. Aladekomo, Photochem. Photobiol. 2 (1965) 415.

[9] B. Stcvens, Spectrochim. Acta 18 (1962) 439.

[10] J.B. Birks and A.A. Kazzaz, Proc. Roy. Soc. A 304 (1968) 291.

111 G. Castro and R.M. Hochstrasser, J. Chem. Phys. 45 (1966) 4252.

[12] E.C. Lim and S.K. Chakrabarti, Mol. Phys. 13 (1967) 293.
[13] D.J. Cram, N.L. Allinger and H. Steinberg, J. Am. Chem. Soc. 76 (1954) 6132.

[14] H.H. Wassermann and P.M. Keehn, J. Am. Chem. Soc. 91 (1969) 2374.

[15] H.A. Staab and M. Haenel, Chem. Ber. 106 (1973) 2190.

[16] M. Haenel and H.A. Staab, Chem. Ber. 106 (1973) 2203.

[17] M.A. El-Sayed, Nature 197 (1963) 481.

[18] M.T. Vala, J. Haebig and S.A. Rice, J. Chem. Phys. 43 (1965) 8 B6.

[19] M.T. Vala, I.H. Hillier, S.A. Rice and J. Jortner, J. Chem. Phys. 44 (1966) 23.

[20] I.H. Hillier, L. Glass and S.A. Rice, J. Chem. Phys. 45 (1966) 3015; J. Am. Chem. Soc. 88 (1966) 5063.

[21] S. Iwata, K. Fuke, M. Sasaki, S. Nagakura, T. Otsubo, and S. Misumi, J. Mol. Spectry. 46 (1973) 1.

[22] K. Fuke and S. Nagakura, Bul. Chem. Soc. Japan 48 (1975) 46.

[23] J.R. Froines and P.J. Hagermann, Chem. Phys. Letters 4 (1969) 195.

[24] F. B. Bramwell and J. Gendell, J. Chem. Phys. 58 (1973) 420.

[25] M. Haenel, Thesis, Ruprecht-Karl-Universität, Heidelberg (1971).

[26] S. Iwata, J. Tanaka and S. Nagakura, J. Chem. Phys. 47 (1967) 2203.

[27] J.P. Colpa and M.F.J. Islip, Mol. Phys. 25 (1973) 701.

[28] J.P. Colpa and F.E. Brown, Mol. Phys. 26 (1973) 1453.

[29] J.P. Colpa, Mol. Phys. 28 (1974) 581.

[30] J.P. Colpa, A.j. Thakkar, V.H. Smith and P. Randle, Mol. Phys. 29 (1975) 1861.

[31] E.A. Colbourn, J. Phys, B 6 (1973) 2618.

[32] T.H. Dunning, W.J. Hunt and W.A. Goddard, Chem. Phys. Letters 4 (1969) 147.

C.F. Bender, T.H. Dunning, H.F. Schaefer, W.A. Goddard and W.J. Hunt, Chem. Phys. Letters 15 (1972) 171.

[33] M. Jungen, Chem. Phys. Letters 27 (1974) 256.

[34] R.P. Hosteny, T.H. Dunning Jr., R.R. Gilman and A. Pipano, J. Chem. Phys. 62 (1975) 4764.

[35] A. Bree and R. Zwarich, J. Chem. Phys. 51 (1969) 903;

[36] J. Behnke, Thesis, Ruprecht-Karl-Universität, Heidelberg (1975).

[37] C.J. Winscom and A.H. Maki, Chem. Phys. Letters 12 (1971) 264.

[38] D. Schweitzer, J. Zuclich and A.H. Maki, Mol. Phys. 25 (1973) 193.

[39] J. Zuclich, D. Schweitzer and A.H. Maki, Photochem. Photobiol. 18 (1973) 161.

[40] W.E. Graves, R.H. Hofeldt and S.P. MoGlynn; J. Chem. Phys. 56 (1972) 1309.

[41] D. Schweitzer, K.H. Hausser, V. Taglieber and H.A. Staab, to be published.

[42] D. Haarer, Chem. Phys. Letters 27 (1974) 91. 